\title{
Adiabatic and entropy perturbations with interacting fluids and fields
}

\author{
Karim A. Malik ${ }^{1}$ and David Wands ${ }^{2}$ \\ ${ }^{1}$ Physics Department, University of Lancaster, Lancaster LA1 4YB, United Kingdom \\ ${ }^{2}$ Institute of Cosmology and Gravitation, University of Portsmouth, Portsmouth PO1 2EG, United Kingdom
}

(Dated: February 2, 2008)

\begin{abstract}
We develop a gauge-invariant formalism for the study of density perturbations in a FriedmannRobertson-Walker universe with multiple interacting fluids and/or scalar fields. We show how $N$ scalar fields may be described by $N$ kinetic fluids (with maximally stiff equation of state) interacting with a non-dynamical potential (with vacuum equation of state). We split generic perturbations into adiabatic and entropic parts, and give the coupled first-order evolution equations on all scales, including energy and momentum exchange. We identify the non-adiabatic effects on large scales, and define adiabatic initial conditions in the presence of multiple fluids and fields.
\end{abstract}

PACS numbers: $98.80 . \mathrm{Cq}$

JCAP02 (2005) 007, astro-ph/0411703 v2

\section{INTRODUCTION}

The large-scale structure of the cosmos provides the key to understanding the early evolution of our Universe. In particular primordial density perturbations may be produced from quantum fluctuations during an inflationary era. Most gauge-invariant studies of linear perturbations about homogeneous and isotropic cosmological background solution follow from the seminal article of Bardeen [1]. In particular the reviews of Kodama and Sasaki 2] and Mukhanov, Feldman and Brandenberger [3] have become widely used reference works. An alternative "covariant" approach has been advocated by Ellis and collaborators [4] and developed by Challinor and Lasenby [5]. These works have provided gauge-invariant descriptions of the evolution of the total density, pressure and velocity perturbations in various cosmological models.

Recently there has been increasing emphasis on the evolution of perturbations in multi-component systems where it is useful to identify gauge-invariant adiabatic and entropy modes. This is partly driven by more detailed models of the multi-component systems in the early universe, such as reheating at the end of inflation [6], or the effect of late-decaying scalar fields [7]. But it is also needed to make more detailed comparison with observational data in the late-time universe containing radiation, matter and dark energy (see, e.g., [8]).

On large scales, where the perturbed universe can be treated as locally homogeneous and isotropic, we can identify a conserved perturbation with any locally conserved quantity [9]. In particular there is a conserved perturbation associated with any perfect fluid whose energy density is locally conserved 10. This can be generalised to adiabatic perturbations of a multi-component cosmology and where the conserved perturbation is the gauge-invariant curvature perturbation on uniform total density hypersurfaces, $\zeta$, introduced by Bardeen and collaborators [11, 12].

Conversely, $\zeta$ is not in general conserved on large scales when one considers non-adiabatic perturbations of a multicomponent system. Nonetheless, at any instant, it is possible to decompose arbitrary perturbations of the multicomponent cosmology into an adiabatic mode, with an associated conserved perturbation on large scales, and entropy (or isocurvature) modes which evolve independently of the adiabatic mode on large scales. One consequence of the effect of non-adiabatic perturbations upon the evolution of the total curvature perturbation, $\zeta$, can be the generation of correlations between the primordial curvature perturbation and any surviving isocurvature perturbations at the time of last-scattering of the cosmic microwave background [13].

In this paper we will develop the gauge-invariant theory of cosmological perturbations to deal with multiple interacting fluids and fields. We develop the multi-component formalism of Kodama and Sasaki [2] (see also [14]). We subtly redefine the relative entropy perturbations to ensure gauge-invariance when including energy transfer between fluids. The resulting simplified evolution equations in the large-scale limit were presented in Ref. 15. We also relate this fluid description to previous work identifying adiabatic and entropy modes in multiple scalar field systems 16] (see also Refs. 17, 18, 19, 20, 21]). Hwang has also investigated the evolution of perturbations with multiple fluids [22]. See Refs. 23] for an alternative long-wavelength approximation.

The paper is organised as follows. In Section 1 we introduce the governing equations with multiple interacting fluids in the homogeneous background and for first-order inhomogeneous perturbations on all scales. In Section [II] we identify gauge-invariant perturbations describing the total density, velocity and shear perturbations and the relative entropy and velocity perturbations between different components, allowing for energy and momentum transfer between the fluids. We give the coupled first-order evolution equations and in particular give the simplified set of evolution equations valid in the large-scale limit. Including interactions enables us to treat fluids and scalar fields on the same footing and we show in Section IV how $N$ scalar fields can be described as $N$ "kinetic fluids" interacting with one 
"potential fluid" describing the vacuum energy. We conclude in the final section with a short discussion.

In Appendix $\mathrm{A}$ we present the gauge transformation properties of the metric and matter variables including energy and momentum transfer and give some identities relating different variables that prove useful in simplifying our equations. In Appendix B we give the governing equations on all scales in the uniform curvature gauge which may be better suited to numerical solutions.

Throughout this work we assume a flat Friedmann-Robertson-Walker (FRW) background spacetime. Greek indices, $\mu, \nu, \lambda$, run from $0, \ldots 3$, while lower case Latin indices, $i, j, k$, run from $1, \ldots 3$. Greek indices from the beginning of the alphabet, $\alpha, \beta, \gamma$ will be used to denote different fluids, and upper case Latin indices, $I, J, K$, denote different scalar fields. We only consider linear scalar perturbations in this article and leave vector and tensor perturbations to a future publication [24].

\section{GOVERNING EQUATIONS}

In this section we give the governing equations for a system of multiple interacting fluids.

The covariant Einstein equations are given by

$$
G_{\mu \nu}=8 \pi G T_{\mu \nu}
$$

where $G_{\mu \nu}$ is the Einstein tensor, $T_{\mu \nu}$ is the total energy-momentum tensor, and $G$ is Newton's constant. Through the Bianchi identities, the field equations (2.1) imply the local conservation the total energy and momentum,

$$
\nabla_{\mu} T^{\mu \nu}=0 .
$$

In the multiple fluid case the total energy-momentum tensor is the sum of the energy-momentum tensors of the individual fluids

$$
T^{\mu \nu}=\sum_{\alpha} T_{(\alpha)}^{\mu \nu}
$$

For each fluid we write the local energy-momentum transfer 4-vector as

$$
\nabla_{\mu} T_{(\alpha)}^{\mu \nu}=Q_{(\alpha)}^{\nu}
$$

where energy-momentum is locally conserved, $Q_{(\alpha)}^{\nu}=0$, only for non-interacting fluids. Equations (2.2) and (2.4) imply the constraint

$$
\sum_{\alpha} Q_{(\alpha)}^{\nu}=0
$$

\section{A. Background equations}

The Einstein equations (2.1) give the Friedmann constraint and evolution equation for the background FRW universe

$$
\begin{aligned}
H^{2} & =\frac{8 \pi G}{3} \rho, \\
\dot{H} & =-4 \pi G(\rho+P),
\end{aligned}
$$

and energy-momentum conservation, Eq. (2.2), gives the continuity equation

$$
\dot{\rho}=-3 H(\rho+P),
$$

where $\rho$ and $P$ are the total energy density and the total pressure, a dot denotes a derivative with respect to coordinate time, $t$, the scale factor is $a=a(t)$, and $H \equiv \dot{a} / a$ is the Hubble parameter.

The total density and the total pressure are related to the density and pressure of the component fluids by

$$
\sum_{\alpha} \rho_{\alpha}=\rho, \quad \sum_{\alpha} P_{\alpha}=P .
$$


The continuity equation (2.4) for each individual fluid in the background is [2]

$$
\dot{\rho}_{\alpha}=-3 H\left(\rho_{\alpha}+P_{\alpha}\right)+Q_{\alpha}
$$

where the energy transfer to the $\alpha$-fluid is given by the time component of the energy-momentum transfer vector $Q_{(\alpha)}^{0}=Q_{\alpha}$ in the background. Equation (2.5) implies that the background energy transfer obeys the constraint

$$
\sum_{\alpha} Q_{\alpha}=0
$$

\section{B. Perturbed equations}

We will consider linear scalar perturbations about a spatially-flat FRW background model, defined by the line element

$$
d s^{2}=-(1+2 \phi) d t^{2}+2 a B_{, i} d t d x^{i}+a^{2}\left[(1-2 \psi) \delta_{i j}+2 E_{, i j}\right] d x^{i} d x^{j}
$$

where we use the notation of Ref. [3] for the gauge-dependent curvature perturbation, $\psi$, the lapse function, $\phi$, and scalar shear, $\sigma_{\mathrm{s}} \equiv a^{2} \dot{E}-a B$, and where $\delta_{i j}$ denotes the flat background metric and $X_{, i} \equiv \partial X / \partial x^{i}$. The transformation of these scalar metric perturbations under arbitrary gauge-transformations is given in Appendix A 1

\section{Energy and momentum conservation}

The perturbed energy transfer 4-vector, Eq. (2.4), including terms up to first order, is written as [2, 15]

$$
\begin{aligned}
Q_{(\alpha) 0} & =-Q_{\alpha}(1+\phi)-\delta Q_{\alpha} \\
Q_{(\alpha) i} & =\left(f_{\alpha}+Q_{\alpha} V\right)_{, i}
\end{aligned}
$$

and Eq. 2.5) implies that the perturbed energy and momentum transfer obey the constraints

$$
\sum_{\alpha} \delta Q_{\alpha}=0, \quad \sum_{\alpha} f_{\alpha}=0
$$

The perturbed energy conservation equation for a particular fluid, including energy transfer, is then obtained by the first-order part of the time-component of the perturbed continuity equation (2.4) to give [2, 25]

$$
\dot{\delta \rho_{\alpha}}+3 H\left(\delta \rho_{\alpha}+\delta P_{\alpha}\right)-3\left(\rho_{\alpha}+P_{\alpha}\right) \dot{\psi}+\frac{\nabla^{2}}{a^{2}}\left(\rho_{\alpha}+P_{\alpha}\right)\left(V_{\alpha}+\sigma_{\mathrm{s}}\right)-Q_{\alpha} \phi-\delta Q_{\alpha}=0,
$$

where $\delta \rho_{\alpha}$ and $\delta P_{\alpha}$ are the perturbed energy density and the perturbed pressure of the $\alpha$-fluid, respectively, and the comoving spatial Laplacian is denoted by $\nabla^{2} \equiv \delta^{i j} \partial^{2} / \partial x^{i} \partial x^{j} . V_{\alpha}$ is the covariant velocity perturbation of the $\alpha$-fluid defined as

$$
V_{\alpha} \equiv a\left(v_{\alpha}+B\right)
$$

where $v_{\alpha}$ is the scalar velocity potential of the $\alpha$-fluid. The momentum conservation equation of the $\alpha$-fluid is

$$
\dot{V}_{\alpha}+\left[\frac{Q_{\alpha}}{\rho_{\alpha}+P_{\alpha}}\left(1+c_{\alpha}^{2}\right)-3 H c_{\alpha}^{2}\right] V_{\alpha}+\phi+\frac{1}{\rho_{\alpha}+P_{\alpha}}\left[\delta P_{\alpha}+\frac{2}{3} \frac{\nabla^{2}}{a^{2}} \Pi_{\alpha}-Q_{\alpha} V-f_{\alpha}\right]=0,
$$

where $\Pi_{\alpha}$ is the perturbed scalar anisotropic stress of the $\alpha$-fluid and $c_{\alpha}^{2} \equiv \dot{P}_{\alpha} / \dot{\rho}_{\alpha}$ is the adiabatic sound speed of that fluid. Note that for stress-free, non-interacting dust we have $\dot{V}_{\alpha}+\phi=0$ and hence $V_{\alpha}=$ constant in a synchronous gauge, whereas the scalar velocity potential redshifts as $v_{\alpha} \propto 1 / a$. In Ref. [15] we used $\delta q_{\alpha} \equiv\left(\rho_{\alpha}+P_{\alpha}\right) V_{\alpha}$ as the momentum perturbation.

The total fluid perturbations are related to the individual fluid quantities by

$$
\sum_{\alpha} \delta \rho_{\alpha}=\delta \rho, \quad \sum_{\alpha} \delta P_{\alpha}=\delta P, \quad \sum_{\alpha} \Pi_{\alpha}=\Pi
$$


and

$$
V=\sum_{\gamma} \frac{\rho_{\gamma}+P_{\gamma}}{\rho+P} V_{\gamma}
$$

where $\delta \rho$ is the total density perturbation, $\delta P$ the total pressure perturbation, $\Pi$ the total anisotropic stress, and the total covariant velocity perturbation is given by

$$
V \equiv a(v+B)
$$

where $v$ is the total scalar velocity potential.

We therefore get an evolution equation for the total density perturbation from Eq. (2.15) by summing over all fluids, using Eq. (2.18) and the constraint (2.14),

$$
\dot{\delta \rho}+3 H(\delta \rho+\delta P)-3(\rho+P) \dot{\psi}+\frac{\nabla^{2}}{a^{2}}(\rho+P)\left(V+\sigma_{\mathrm{s}}\right)=0
$$

while the total momentum conservation equation is given from Eq. (2.17), using Eqs. (2.18) and (2.19) and the constraint (2.14),

$$
\dot{V}-3 H c_{\mathrm{s}}^{2} V+\phi+\frac{1}{\rho+P}\left(\delta P+\frac{2}{3} \frac{\nabla^{2}}{a^{2}} \Pi\right)=0,
$$

where $c_{\mathrm{s}}^{2}$ is the adiabatic speed of sound, defined as

$$
c_{\mathrm{s}}^{2} \equiv \frac{\dot{P}}{\dot{\rho}} .
$$

\section{Einstein's field equations} 25]

The $G_{0}^{0}$ component of the Einstein equations (2.1) yields the first-order perturbed energy constraint equation [2, 3 ,

$$
3 H(\dot{\psi}+H \phi)-\frac{\nabla^{2}}{a^{2}}\left(\psi+H \sigma_{\mathrm{s}}\right)+4 \pi G \delta \rho=0 .
$$

We get the momentum constraint equation (identically zero in the FRW background) from the $G_{i}^{0}$-component of the Einstein equations,

$$
\dot{\psi}+H \phi+4 \pi G(\rho+P) V=0 .
$$

From the trace-free part of the $G_{j}^{i}$ component of Einstein's equations we find the shear evolution equation

$$
\dot{\sigma}_{\mathrm{s}}+H \sigma_{\mathrm{s}}-\phi+\psi-8 \pi G \Pi=0,
$$

and the trace of the $G_{j}^{i}$ component of Einstein's equations gives, using Eq. (2.26), the evolution equation for the curvature perturbation as

$$
\ddot{\psi}+3 H \dot{\psi}+H \dot{\phi}+\left(3 H^{2}+2 \dot{H}\right) \phi-4 \pi G\left(\delta P+\frac{2}{3} \frac{\nabla^{2}}{a^{2}} \Pi\right)=0 .
$$

\section{GAUGE-INVARIANT PERTURBATIONS AND GOVERNING EQUATIONS}

In this section we define gauge-invariant variables, using the transformation properties of the metric and matter perturbations given in Appendix A 1. We then use the results of Section $\amalg B$ to get the evolution equations for these gauge-invariant variables.

In this and the following sections we will replace spatial Laplacians by the wavenumbers of their respective eigenmodes according to $\nabla^{2} \rightarrow-k^{2}$. 


\section{A. Definitions}

\section{Curvature perturbations}

A gauge-invariant definition of the curvature perturbation for each fluid is given by [10]

$$
\zeta_{\alpha} \equiv-\psi-H \frac{\delta \rho_{\alpha}}{\dot{\rho}_{\alpha}}
$$

This describes the dimensionless density perturbation on uniform-curvature hypersurfaces, or, equivalently at firstorder, the curvature perturbation on uniform $\alpha$-fluid density hypersurfaces [25]. The total curvature perturbation on uniform density hypersurfaces $10,11,12$

$$
\zeta \equiv-\psi-H \frac{\delta \rho}{\dot{\rho}}
$$

is then a weighted sum of the individual fluid perturbations

$$
\zeta=\sum_{\alpha} \frac{\dot{\rho}_{\alpha}}{\dot{\rho}} \zeta_{\alpha}
$$

We can also define a gauge-invariant combination of the curvature and velocity perturbations for each fluid

$$
\mathcal{R}_{\alpha} \equiv \psi-H V_{\alpha}
$$

This describes the curvature perturbation on hypersurfaces orthogonal to worldlines comoving with the fluid, and is particularly useful when studying scalar field perturbations. The total comoving curvature perturbation is given by [26, 27]

$$
\mathcal{R} \equiv \psi-H V,
$$

and is again a weighted sum, using Eq. (2.19), of the individual fluid perturbations

$$
\mathcal{R}=\sum_{\alpha} \frac{\rho_{\alpha}+P_{\alpha}}{\rho+P} \mathcal{R}_{\alpha}
$$

The two alternative descriptions of the total curvature perturbation are closely related. Indeed even the coefficients $\dot{\rho}_{\alpha} / \dot{\rho}$ appearing in Eq. (3.3) and $\left(\rho_{\alpha}+P_{\alpha}\right) /(\rho+P)$ in Eq. (3.6) are the same for non-interacting fluids, i.e., when $Q_{\alpha}=0$. The constraint equation (2.24) can be rewritten in terms of these gauge-invariant quantities as

$$
\frac{k^{2}}{a^{2}} \Psi=3 \dot{H}(\mathcal{R}+\zeta)
$$

where we introduce the gauge-invariant curvature perturbation in the longitudinal or zero-shear gauge [1, 3],

$$
\Psi \equiv \psi+H \sigma_{\mathrm{s}}
$$

In practise it is convenient to use only one of $\zeta$ or $\mathcal{R}$ and use $\Psi$ to keep track of $\zeta+\mathcal{R}$, which, as can be seen from (3.7), typically becomes small on super-Hubble scales, i.e., $k^{2} / a^{2} H^{2} \ll 1$.

The evolution equation for $\zeta_{\alpha}$ is given from Eq. (2.15) and using Eq. (2.24)

$$
\begin{aligned}
\dot{\zeta}_{\alpha}= & 3 \frac{H^{2}}{\dot{\rho}_{\alpha}}\left(\delta P_{\alpha}-c_{\alpha}^{2} \delta \rho_{\alpha}\right)-\dot{H} \frac{Q_{\alpha}}{\dot{\rho}_{\alpha}}\left(\frac{\delta \rho_{\alpha}}{\dot{\rho}_{\alpha}}-\frac{\delta \rho}{\dot{\rho}}\right)-\frac{H}{\dot{\rho}_{\alpha}}\left(\delta Q_{\alpha}-\frac{\dot{Q}_{\alpha}}{\dot{\rho}_{\alpha}} \delta \rho_{\alpha}\right) \\
& +\frac{1}{3 H} \frac{k^{2}}{a^{2}}\left[\Psi-\left(1-\frac{Q_{\alpha}}{\dot{\rho}_{\alpha}}\right) \mathcal{R}_{\alpha}\right]
\end{aligned}
$$

where the terms in the first line are gauge-invariant combinations, which we shall deal with in the next subsection on entropy perturbations. The evolution equation for $\mathcal{R}_{\alpha}$ is given from the momentum conservation equation, Eq. (2.17), and using Eq. (2.24)

$$
\begin{aligned}
\dot{\mathcal{R}}_{\alpha}= & \frac{\dot{H}}{H}\left(\mathcal{R}_{\alpha}-\mathcal{R}\right)-\frac{\dot{\rho}_{\alpha}}{\rho_{\alpha}+P_{\alpha}} H c_{\alpha}^{2}\left(\mathcal{R}_{\alpha}+\zeta_{\alpha}\right) \\
& +\frac{H}{\rho_{\alpha}+P \alpha}\left[\delta P_{\alpha}-c_{\alpha}^{2} \delta \rho_{\alpha}-\frac{2}{3} \frac{k^{2}}{a^{2}} \Pi_{\alpha}-f_{\alpha}-Q_{\alpha}\left(V-V_{\alpha}\right)\right] .
\end{aligned}
$$


Finally we note that the curvature perturbations on uniform total density, uniform shear and comoving hypersurfaces, $\zeta, \Psi$ and $\mathcal{R}$, are related to the density, shear and the velocity perturbations on uniform curvature hypersurfaces,

$$
\widetilde{\delta \rho}=-\frac{\dot{\rho}}{H} \zeta, \quad \tilde{\sigma_{\mathrm{s}}}=\frac{\Psi}{H}, \quad \tilde{V}=-\frac{\mathcal{R}}{H},
$$

and similarly for the curvature perturbations on uniform $\alpha$-density and uniform $\alpha$-velocity hypersurfaces

$$
\widetilde{\delta \rho_{\alpha}}=-\frac{\dot{\rho}}{H} \zeta_{\alpha}, \quad \tilde{V}_{\alpha}=-\frac{\mathcal{R}_{\alpha}}{H},
$$

where the "tilde" denotes perturbations on uniform curvature hypersurfaces. We can therefore also think of the curvature perturbations $\zeta$ and $\zeta_{\alpha}$ as describing density perturbations or density contrasts, of the curvature perturbations $\mathcal{R}$ and $\mathcal{R}_{\alpha}$ as describing velocities, and of the curvature perturbation $\Psi$ as describing the shear.

Governing equations for the variables defined in Eqs. (3.11) and and (3.12) in uniform curvature gauge are given in Appendix B

\section{Entropy perturbations}

The difference between the density perturbation (3.1) for any two fluids describes a gauge-invariant relative entropy (or isocurvature) perturbation 10,15

$$
\mathcal{S}_{\alpha \beta} \equiv 3\left(\zeta_{\alpha}-\zeta_{\beta}\right)=-3 H\left(\frac{\delta \rho_{\alpha}}{\dot{\rho}_{\alpha}}-\frac{\delta \rho_{\beta}}{\dot{\rho}_{\beta}}\right) .
$$

Note that this only coincides with the entropy perturbation defined by Kodama and Sasaki [2] in the absence of energy transfer, $Q_{\alpha}=Q_{\beta}=0$. The factor of 3 is introduced to coincide with the conventional definition of entropy perturbations in the baryon-photon ratio:

$$
\frac{\delta\left(n_{\mathrm{B}} / n_{\gamma}\right)}{n_{\mathrm{B}} / n_{\gamma}}=\mathcal{S}_{\mathrm{B} \gamma}
$$

where $n_{\mathrm{B}}$ and $n_{\gamma}$ are the baryon and the photon number densities, respectively.

Similarly we define the gauge-invariant relative velocity perturbation by $[2]{ }^{1}$

$$
V_{\alpha \beta} \equiv V_{\alpha}-V_{\beta}=-\frac{1}{H}\left(\mathcal{R}_{\alpha}-\mathcal{R}_{\beta}\right) .
$$

The total pressure perturbation can be split into an adiabatic and non-adiabatic part

$$
\delta P \equiv \delta P_{\text {nad }}+c_{\mathrm{s}}^{2} \delta \rho,
$$

where $c_{\mathrm{s}}^{2}$ is the adiabatic speed of sound defined in Eq. (2.23) above.

In the presence of more than one fluid, the total non-adiabatic pressure perturbation, $\delta P_{\text {nad }}$, may be further split into two parts [2],

$$
\delta P_{\text {nad }} \equiv \delta P_{\text {intr }}+\delta P_{\text {rel }} .
$$

The first part is due to the intrinsic entropy perturbation of each fluid

$$
\delta P_{\text {intr }}=\sum_{\alpha} \delta P_{\text {intr }, \alpha},
$$

\footnotetext{
${ }^{1}$ The definition of the relative velocity perturbation in [2] is related to our definition in Eq. [3.16] by$$
V_{\alpha \beta}=a V_{\alpha \beta} \mathrm{KS} .
$$ 
where the intrinsic non-adiabatic pressure perturbation of each fluid is given by

$$
\delta P_{\text {intr }, \alpha} \equiv \delta P_{\alpha}-c_{\alpha}^{2} \delta \rho_{\alpha} .
$$

For any fluid with a definite equation of state, $P_{\alpha}=P_{\alpha}\left(\rho_{\alpha}\right)$, the intrinsic non-adiabatic pressure perturbation must vanish, $\delta P_{\mathrm{intr}, \alpha}=0$. The total adiabatic sound speed (2.23) is the weighted sum of the adiabatic sound speeds of the individual fluids,

$$
c_{\mathrm{s}}^{2}=\sum_{\alpha} \frac{\dot{\rho}_{\alpha}}{\dot{\rho}} c_{\alpha}^{2}
$$

The second part of the non-adiabatic pressure perturbation (3.18) is due to the relative entropy perturbation $\mathcal{S}_{\alpha \beta}$ between different fluids, defined in Eq. (3.13),

$$
\delta P_{\mathrm{rel}}=-\frac{1}{6 H \dot{\rho}} \sum_{\alpha, \beta} \dot{\rho}_{\alpha} \dot{\rho}_{\beta}\left(c_{\alpha}^{2}-c_{\beta}^{2}\right) \mathcal{S}_{\alpha \beta} .
$$

Analogous to the non-adiabatic pressure perturbation for each fluid (3.20), we can identify an intrinsic non-adiabatic part of the energy transfer perturbation [15] that appears in the perturbed continuity equation for each fluid (2.15)

$$
\delta Q_{\mathrm{intr}, \alpha} \equiv \delta Q_{\alpha}-\frac{\dot{Q}_{\alpha}}{\dot{\rho}_{\alpha}} \delta \rho_{\alpha} .
$$

This is automatically zero if the local energy transfer $Q_{\alpha}$ is a function of the local density $\rho_{\alpha}$ so that $\delta Q_{\alpha}=$ $\left(\dot{Q}_{\alpha} / \dot{\rho}_{\alpha}\right) \delta \rho_{\alpha}$, just as the intrinsic non-adiabatic pressure perturbation (3.20) vanishes when $\delta P_{\alpha}=\left(\dot{P}_{\alpha} / \dot{\rho}_{\alpha}\right) \delta \rho_{\alpha}$. Note however, that from the definition, Eq. (3.23), the intrinsic non-adiabatic energy transfer perturbation, $\delta Q_{\text {intr }, \alpha}$, can be non-zero in the case where the background energy transfer $Q_{\alpha} \neq 0$, even if $\delta Q_{\alpha}=0$. We can also identify a relative non-adiabatic energy transfer [15] that appears in Eq. (2.15) whenever $Q_{\alpha} \neq 0$

$$
\delta Q_{\mathrm{rel}, \alpha} \equiv Q_{\alpha} \frac{\dot{H}}{H}\left(\frac{\delta \rho_{\alpha}}{\dot{\rho}_{\alpha}}-\frac{\delta \rho}{\dot{\rho}}\right)=-\frac{Q_{\alpha}}{6 H \rho} \sum_{\beta} \dot{\rho}_{\beta} \mathcal{S}_{\alpha \beta},
$$

due to the presence of relative entropy perturbations. Only the intrinsic parts of the non-adiabatic pressure and energy-transfer perturbations will appear in the evolution equation for the relative entropy perturbation, Eq. (3.31) below.

With the above definitions the evolution equation for the curvature perturbation $\zeta_{\alpha}$, Eq. (3.9), reduces to

$$
\dot{\zeta}_{\alpha}=3 \frac{H^{2}}{\dot{\rho}_{\alpha}} \delta P_{\mathrm{intr}, \alpha}-\frac{H}{\dot{\rho}_{\alpha}}\left(\delta Q_{\mathrm{intr}, \alpha}+\delta Q_{\mathrm{rel}, \alpha}\right)+\frac{1}{3 H} \frac{k^{2}}{a^{2}}\left[\Psi-\left(1-\frac{Q_{\alpha}}{\dot{\rho}_{\alpha}}\right) \mathcal{R}_{\alpha}\right] .
$$

Thus we see that $\dot{\zeta}_{\alpha} \simeq 0$ in the absence of non-adiabatic pressure and energy transfer perturbations, on the large scales where we can neglect gradient terms.

There is no momentum transfer in the background FRW universe so the momentum transfer $f_{\alpha}$ that appears in the momentum conservation equation for each fluid (2.17) is automatically gauge-invariant. Nevertheless we can identify a relative momentum transfer in Eq. (2.17), defined as

$$
f_{\mathrm{rel}, \alpha} \equiv Q_{\alpha}\left(V-V_{\alpha}\right),
$$

which can be rewritten in terms of the relative velocity perturbation, defined in Eq. (3.16), using Eq. (A24), as

$$
f_{\mathrm{rel}, \alpha}=-Q_{\alpha} \sum_{\gamma} \frac{\rho_{\gamma}+P_{\gamma}}{\rho+P} V_{\alpha \gamma}
$$

The evolution equation for $\mathcal{R}_{\alpha}$, Eq. (3.10) then simplifies to

$$
\dot{\mathcal{R}}_{\alpha}=\frac{\dot{H}}{H}\left(\mathcal{R}_{\alpha}-\mathcal{R}\right)-\frac{\dot{\rho}_{\alpha}}{\rho_{\alpha}+P \alpha} H c_{\alpha}^{2}\left(\mathcal{R}_{\alpha}+\zeta_{\alpha}\right)+\frac{H}{\rho_{\alpha}+P \alpha}\left[\delta P_{\mathrm{intr}, \alpha}-\frac{2}{3} \frac{k^{2}}{a^{2}} \Pi_{\alpha}-f_{\alpha}-f_{\mathrm{rel}, \alpha}\right] .
$$

We end up with the following set of gauge-invariant dynamical variables: the curvature perturbation on uniform density hypersurfaces, $\zeta$, the curvature perturbation on uniform shear hypersurfaces $\Psi$, the relative entropy perturbation $\mathcal{S}_{\alpha \beta}$, and the relative velocity perturbation $V_{\alpha \beta}$. 


\section{B. Evolution equations}

In this sub-section we re-express the gauge dependent evolution equations given in Section IIB in terms of the gauge-invariant quantities defined above in Section ஹA In deriving the evolution equations, we make extensive use of the relation between the relative entropy perturbation and the $\alpha$-fluid and total density perturbations, Eq. A23, and the relation between the relative velocity perturbation and the $\alpha$-fluid and total velocity perturbations, Eq. (A24).

The first-order evolution equation for the total curvature perturbation, $\zeta$, defined in Eq. (3.2), derives from the evolution equation of the total density perturbation, Eq. (2.21), and is given by

$$
\dot{\zeta}=-\frac{H}{\rho+P} \delta P_{\text {nad }}+\frac{1}{3 H} \frac{k^{2}}{a^{2}}(\Psi-\zeta)+\frac{1}{9 H \dot{H}} \frac{k^{4}}{a^{4}} \Psi,
$$

where $\delta P_{\text {nad }}$, as defined in Eq. (3.18) depends on the intrinsic entropy perturbations, $\delta P_{\text {intr }, \alpha}$ defined in Eq. (3.20), and the relative entropy perturbations, $\mathcal{S}_{\alpha \beta}$ defined in Eq. (3.13).

The evolution equation for the curvature perturbation in the zero-shear gauge, $\Psi$, defined in Eq. (3.8), follows from Eqs. (2.25) and (2.26)

$$
\dot{\Psi}+\left(H-\frac{\dot{H}}{H}\right) \Psi+\frac{1}{3 H} \frac{k^{2}}{a^{2}} \Psi-\frac{\dot{H}}{H} \zeta=-8 \pi G H \Pi .
$$

Note that energy-momentum transfer between individual fluids does not directly enter into the evolution equations for the total curvature perturbations $\zeta$ and $\Psi$. However it does considerably complicate the evolution equations for the relative entropy and relative velocity perturbations, as can be seen below.

The evolution of the relative entropy perturbation, $\mathcal{S}_{\alpha \beta}$ defined in Eq. (3.13), using Eq. (2.15), is given by

$$
\begin{aligned}
\dot{\mathcal{S}}_{\alpha \beta}= & 3 H\left(\frac{3 H \delta P_{\text {intr }, \alpha}-\delta Q_{\text {intr }, \alpha}}{\dot{\rho}_{\alpha}}-\frac{3 H \delta P_{\text {intr }, \beta}-\delta Q_{\text {intr }, \beta}}{\dot{\rho}_{\beta}}\right) \\
& +\frac{\dot{H}}{2 H}\left[\left(\frac{Q_{\alpha}}{\dot{\rho}_{\alpha}}+\frac{Q_{\beta}}{\dot{\rho}_{\beta}}\right) \mathcal{S}_{\alpha \beta}+\left(\frac{Q_{\alpha}}{\dot{\rho}_{\alpha}}-\frac{Q_{\beta}}{\dot{\rho}_{\beta}}\right) \sum_{\gamma} \frac{\dot{\rho}_{\gamma}}{\dot{\rho}}\left(\mathcal{S}_{\alpha \gamma}+\mathcal{S}_{\beta \gamma}\right)\right] \\
& +\frac{k^{2}}{a^{2}}\left[\left(1-\frac{Q_{\alpha}}{2 \dot{\rho}_{\alpha}}-\frac{Q_{\beta}}{2 \dot{\rho}_{\beta}}\right) V_{\alpha \beta}-\left(\frac{Q_{\alpha}}{\dot{\rho}_{\alpha}}-\frac{Q_{\beta}}{\dot{\rho}_{\beta}}\right)\left(\frac{1}{H} \zeta+\frac{1}{2} \sum_{\gamma} \frac{\rho_{\gamma}+P_{\gamma}}{\rho+P}\left(V_{\alpha \gamma}+V_{\beta \gamma}\right)\right)\right] \\
& +\frac{k^{4}}{a^{4}} \frac{1}{3 H \dot{H}}\left(\frac{Q_{\alpha}}{\dot{\rho}_{\alpha}}-\frac{Q_{\beta}}{\dot{\rho}_{\beta}}\right) \Psi .
\end{aligned}
$$

Only the intrinsic non-adiabatic perturbations $\delta P_{\text {intr }, \alpha}$ and $\delta Q_{\text {intr, } \alpha}$ appear explicitly in the evolution equation (3.31), while the relative part of the non-adiabatic energy transfer perturbations (3.24) have been expressed in terms of $\mathcal{S}_{\alpha \beta}$.

Finally, using the evolution equation for the velocity perturbation of the $\alpha$-fluid, Eq. (2.17), we can derive an evolution equation for the gauge-invariant relative velocity perturbation, $V_{\alpha \beta}$ defined in Eq. (3.16),

$$
\begin{aligned}
\dot{V}_{\alpha \beta}+ & \frac{1}{2}\left[\frac{Q_{\alpha}}{\rho_{\alpha}+P_{\alpha}}+\frac{Q_{\beta}}{\rho_{\beta}+P_{\beta}}\right] V_{\alpha \beta}+\frac{1}{2}\left[\frac{Q_{\alpha} c_{\alpha}^{2}}{\rho_{\alpha}+P_{\alpha}}+\frac{Q_{\beta} c_{\beta}^{2}}{\rho_{\beta}+P_{\beta}}-3 H\left(c_{\alpha}^{2}+c_{\beta}^{2}\right)\right]\left(V_{\alpha \beta}-\frac{1}{3 H} \mathcal{S}_{\alpha \beta}\right) \\
+ & \frac{1}{2}\left[\frac{Q_{\alpha}}{\rho_{\alpha}+P_{\alpha}}\left(1+c_{\alpha}^{2}\right)-\frac{Q_{\beta}}{\rho_{\beta}+P_{\beta}}\left(1+c_{\beta}^{2}\right)-3 H\left(c_{\alpha}^{2}-c_{\beta}^{2}\right)\right] \sum_{\gamma} \frac{\rho_{\gamma}+P_{\gamma}}{\rho+P}\left(V_{\alpha \gamma}+V_{\beta \gamma}\right) \\
& -\left[\frac{Q_{\alpha} c_{\alpha}^{2}}{\rho_{\alpha}+P_{\alpha}}-\frac{Q_{\beta} c_{\beta}^{2}}{\rho_{\beta}+P_{\beta}}-3 H\left(c_{\alpha}^{2}-c_{\beta}^{2}\right)\right]\left(\frac{1}{3 H \dot{H}} \frac{k^{2}}{a^{2}} \Psi+\frac{1}{6 H} \sum_{\gamma} \frac{\dot{\rho}_{\gamma}}{\dot{\rho}}\left(\mathcal{S}_{\alpha \gamma}+\mathcal{S}_{\beta \gamma}\right)\right) \\
& -\frac{2}{3} \frac{k^{2}}{a^{2}}\left(\frac{\Pi_{\alpha}}{\rho_{\alpha}+P_{\alpha}}-\frac{\Pi_{\beta}}{\rho_{\beta}+P_{\beta}}\right)-\left(\frac{f_{\alpha}}{\rho_{\alpha}+P_{\alpha}}-\frac{f_{\beta}}{\rho_{\beta}+P_{\beta}}\right)+\left(\frac{\delta P_{\text {intr }, \alpha}}{\rho_{\alpha}+P_{\alpha}}-\frac{\delta P_{\text {intr }, \beta}}{\rho_{\beta}+P_{\beta}}\right)=0
\end{aligned}
$$

where we have used Eq. (3.27) for the relative momentum transfer perturbation.

We therefore see that the evolution equations for $\zeta, \Psi, \mathcal{S}_{\alpha \beta}$ and $V_{\alpha \beta}$, Eqs. (3.29)-(3.32), form a system of coupled, first order, ordinary differential equations. Although we have defined $n(n-1) / 2$ relative entropy perturbations, $\mathcal{S}_{\alpha \beta}$, for $n$ fluids, there are of course only $n-1$ independent entropy perturbations, and similarly $n-1$ independent relative velocity perturbations. Thus we have $2 n$ coupled equations relating $2 n$ variables describing the density and velocity perturbations in $n$ fluids. 
The system is not closed until we specify the intrinsic non-adiabatic pressure perturbation $\delta P_{\text {intr }, \alpha}$, the intrinsic nonadiabatic energy transfer perturbation $\delta Q_{\mathrm{intr}, \alpha}$, the anisotropic stresses $\Pi_{\alpha}$ and the perturbed momentum transfer $f_{\alpha}$ for each fluid. In the background we have to prescribe the equation of state, $P_{\alpha}\left(\rho_{\alpha}\right)$, which determines the adiabatic sound speed $c_{\alpha}^{2}$, and the energy transfer $Q_{\alpha}$. All these quantities depend upon the physical model for the fluids. For perfect fluids $\delta P_{\text {intr, } \alpha}, \delta Q_{\text {intr, } \alpha}, \Pi_{\alpha}$, and $f_{\alpha}$ are all zero. In Section IV we will calculate these quantities explicitly for the particular example of $N$ scalar fields.

\section{Large-scale limit}

Throughout much of the early history of our Universe, scales of astrophysical interest are far larger than the Hubble scale, $H^{-1}$, which defines the cosmological expansion time. For instance, the Hubble scale at the epoch of primordial nucleosynthesis corresponds to a comoving scale of around $10 \mathrm{pc}$ in the present Universe. Thus it is often a very good approximation to work in a large scale limit when studying the origin and evolution in the early universe of the large scale structure of our observable Universe. In this section we give the governing equations in the large scale limit, i.e. in the limit $k \rightarrow 0$. Some of the results in this section have already been presented in [15].

The form of the evolution equations for the perturbations suggests that the time-dependent amplitude of any perturbation, $\delta X(t) e^{i \mathbf{k} \cdot \mathbf{x}}$, can be expanded as a Taylor series in terms of the comoving wavenumber, $k$, as

$$
\delta X(t) \equiv \sum_{n=0,1, \ldots}^{\infty} k^{2 n} \delta X^{(n)}(t) .
$$

Equations (3.29)-(3.32) then give coupled first-order evolution equations for $X_{n}(t)$ driven by $X_{n-1}(t)$ for $n \geq 1$ [and driven by $X_{n-2}$ for $n \geq 2$ in Eqs. (3.29) and (3.31)].

The long-wavelength limit as $k \rightarrow 0$ is given by the time-dependence of the $n=0$ solutions to the homogeneous set of first-order equations:

$$
\begin{aligned}
\dot{\zeta}^{(0)}= & -\frac{H}{\rho+P} \delta P_{\text {nad }}^{(0)} \\
\dot{\Psi}^{(0)}= & -\left(H-\frac{\dot{H}}{H}\right) \Psi^{(0)}+\frac{\dot{H}}{H} \zeta^{(0)}-8 \pi G H \Pi^{(0)}, \\
\dot{\mathcal{S}}_{\alpha \beta}^{(0)}= & 3 H\left(\frac{3 H \delta P_{\mathrm{intr}, \alpha}^{(0)}-\delta Q_{\mathrm{intr}, \alpha}^{(0)}}{\dot{\rho}_{\alpha}}-\frac{3 H \delta P_{\mathrm{intr}, \beta}^{(0)}-\delta Q_{\mathrm{intr}, \beta}^{(0)}}{\dot{\rho}_{\beta}}\right)+\sum_{\gamma} \frac{\dot{\rho}_{\gamma}}{2 \rho}\left(\frac{Q_{\alpha}}{\dot{\rho}_{\alpha}} \mathcal{S}_{\alpha \gamma}^{(0)}-\frac{Q_{\beta}}{\dot{\rho}_{\beta}} \mathcal{S}_{\beta \gamma}^{(0)}\right), \\
\dot{V}_{\alpha \beta}^{(0)}= & -\frac{1}{2}\left[\frac{Q_{\alpha}}{\rho_{\alpha}+P_{\alpha}}+\frac{Q_{\beta}}{\rho_{\beta}+P_{\beta}}\right] V_{\alpha \beta}^{(0)}-\frac{1}{2}\left[\frac{Q_{\alpha} c_{\alpha}^{2}}{\rho_{\alpha}+P_{\alpha}}+\frac{Q_{\beta} c_{\beta}^{2}}{\rho_{\beta}+P_{\beta}}-3 H\left(c_{\alpha}^{2}+c_{\beta}^{2}\right)\right]\left(V_{\alpha \beta}^{(0)}-\frac{1}{3 H} \mathcal{S}_{\alpha \beta}^{(0)}\right) \\
& -\frac{1}{2}\left[\frac{Q_{\alpha}}{\rho_{\alpha}+P_{\alpha}}\left(1+c_{\alpha}^{2}\right)-\frac{Q_{\beta}}{\rho_{\beta}+P_{\beta}}\left(1+c_{\beta}^{2}\right)-3 H\left(c_{\alpha}^{2}-c_{\beta}^{2}\right)\right] \sum_{\gamma} \frac{\rho_{\gamma}+P_{\gamma}}{\rho+P}\left(V_{\alpha \gamma}^{(0)}+V_{\beta \gamma}^{(0)}\right) \\
& +\left[\frac{Q_{\alpha} c_{\alpha}^{2}}{\rho_{\alpha}+P_{\alpha}}-\frac{Q_{\beta} c_{\beta}^{2}}{\rho_{\beta}+P_{\beta}}-3 H\left(c_{\alpha}^{2}-c_{\beta}^{2}\right)\right] \frac{1}{6 H} \sum_{\gamma} \frac{\dot{\rho}_{\gamma}}{\dot{\rho}}\left(\mathcal{S}_{\alpha \gamma}^{(0)}+\mathcal{S}_{\beta \gamma}^{(0)}\right) \\
& +\left(\frac{f_{\alpha}^{(0)}}{\rho_{\alpha}+P_{\alpha}}-\frac{f_{\beta}^{(0)}}{\rho_{\beta}+P_{\beta}}\right)-\left(\frac{\delta P_{\mathrm{intr}, \alpha}^{(0)}}{\rho_{\alpha}+P_{\alpha}}-\frac{\delta P_{\mathrm{intr}, \beta}^{(0)}}{\rho_{\beta}+P_{\beta}}\right) .
\end{aligned}
$$

This long-wavelength limit exists so long as the inhomogeneous source terms in the full evolution equations (3.29)(3.32), that is $\delta P_{\text {intr }, \alpha}, \delta Q_{\text {intr }, \alpha}, f_{\alpha}$ and $\Pi_{\alpha}$, all have a well-defined limit as $k \rightarrow 0$.

The perturbation, $\Psi$, vanishes from the other evolution equations completely in the large scale limit. Since this variable describes the shear (on uniform curvature hypersurfaces), its disappearance can be intuitively understood by observing that the shear is expected to vanish on large scales. Because the shear and velocity perturbations, $\Psi$ and $V_{\alpha \beta}$, only enter the evolution equations (3.29) and (3.31) at order $k^{2}$, we can obtain a long-wavelength limit for for $\zeta^{(0)}$ and $\mathcal{S}_{\alpha \beta}^{(0)}$ under the weaker requirement that $\delta P_{\text {intr, } \alpha}, \delta Q_{\text {intr }, \alpha}, k^{2} f_{\alpha}$ and $k^{2} \Pi_{\alpha}$ all have a well-defined limit as $k \rightarrow 0$. This is the basis of the 'separate universes' picture [10] commonly used to study the evolution of density perturbations on sufficiently large scales where the universe looks locally like an unperturbed (FRW) cosmology. Specifically it requires 
that we can neglect the divergence of the momenta in the zero-shear gauge, $\nabla^{2}\left[\left(\rho_{\alpha}+P_{\alpha}\right)\left(V_{\alpha}+\sigma_{\mathrm{s}}\right)\right]$, in the perturbed continuity equation (2.15) for each fluid.

We see from Eq. (3.34) that in the large scale limit the evolution for the curvature perturbation $\zeta$ is only sourced by the non-adiabatic pressure perturbation, as defined in Eq. (3.18), including contributions from the intrinsic nonadiabatic pressure perturbations of each fluid and the relative entropy perturbations between fluids. For $\delta P_{\text {nad }}=0$ we recover the famous result that $\zeta=$ constant for adiabatic perturbations on large scales 9 , 10, 11, 12, 28, 29]. The long-wavelength solution for adiabatic density perturbations is

$$
\begin{aligned}
\zeta^{(0)} & =C, \\
\Psi^{(0)} & =\frac{H}{a}\left[D+\int a\left(\frac{\dot{H}}{H^{2}} C-8 \pi G \Pi^{(0)}\right) d t\right], \\
\mathcal{S}_{\alpha \beta}^{(0)} & =0 .
\end{aligned}
$$

where $C$ and $D$ are constants of integration. This adiabatic solution exists on large scales even in the presence of energy transfer between fluids, $Q_{\alpha} \neq 0$, so long as the intrinsic non-adiabatic energy transfer, $\delta Q_{\text {intr, } \alpha}$ defined in Eq. (3.23), is zero for each individual fluid.

The evolution of the relative entropy perturbations, $\mathcal{S}_{\alpha \beta}$, is independent of the curvature perturbations $\zeta$ and $\Psi$ in the large-scale limit, Eq. (3.36). The actual evolution is dependent upon the intrinsic non-adiabatic pressure and energy transfer of each fluid. However for perfect fluids all the source terms vanish we have the simple result $\mathcal{S}_{\alpha \beta}=$ constant in the large-scale limit.

The evolution of the relative velocity perturbations $V_{\alpha \beta}$, Eq. (3.37) is driven by relative entropy perturbations, $\mathcal{S}_{\alpha \beta}$, as well as the intrinsic non-adiabatic pressure perturbations and momentum transfer, even on large scales. Only for pressureless, non-interacting fluids do we find $V_{\alpha \beta}=$ constant on large scales.

\section{SCALAR FIELDS}

In this section we show how scalar fields can be included in our interacting fluid formalism. Multiple scalar fields provide a test case in which quantities like the energy transfer and the relative momentum transfer are simple enough to be computed from first principles, and can then be applied to identify adiabatic and entropy modes in the fluid formalism. It also enables us to later "mix" fluids and scalar fields in a consistent way, which is necessary in many cosmological models, including quintessence models of dark energy, decaying scalar fields, such as the curvaton, or inhomogeneous reheating at the end of inflation. In particular we will identify the adiabatic mode and relative entropy perturbations in the fluid description and compare these with previous analyses of adiabatic and entropy modes in the scalar field perturbations [16].

We will split the total energy-momentum of $N$ scalar fields into $N$ maximally stiff fluids interacting with a potential energy. Our splitting is slightly different from that of Hwang and Noh 22] who also relate the scalar field perturbations to fluid quantities. They work in terms of the total density and pressure perturbation for each field, including kinetic and potential perturbations for each field. There is thus an intrinsic non-adiabatic perturbation for each field. By contrast we separate the kinetic and potential perturbations so that each has a fixed equation of state and thus no intrinsic non-adiabatic pressure perturbation and we can always relate non-adiabatic perturbations to relative entropy perturbations between different component parts.

For simplicity we consider here only minimally coupled scalar fields in the sense that they interact only via their combined self-interaction potential. Although we allow for the presence of fluids other than the scalar fields, we assume that the scalar fields do not exchange energy-momentum with any other fluids. 


\section{A. Background}

\section{Standard treatment}

The energy density and the pressure of $N$ minimally coupled scalar fields, labelled by the subscript " $I$ ", are at zeroth order

$$
\begin{aligned}
& \rho_{\varphi}=\frac{1}{2} \sum_{I} \dot{\varphi}_{I}^{2}+U, \\
& P_{\varphi}=\frac{1}{2} \sum_{I} \dot{\varphi}_{I}^{2}-U,
\end{aligned}
$$

where $U=U\left(\varphi_{I}\right)$ is the potential of the scalar fields and upper case Latin indices, $I, J, K$, run from $1, \ldots, N$.

The adiabatic sound speed of all the scalar fields is given from Eq. (2.23) by

$$
c_{\mathrm{s} \varphi}^{2}=1+\frac{2}{3 H} \frac{\dot{U}}{\sum_{I} \dot{\varphi}_{I}^{2}},
$$

where $\dot{U} \equiv \sum_{I} U_{, \varphi_{I}} \dot{\varphi}_{I}$

\section{Splitting into kinetic and potential parts}

We can split the total energy density and pressure of $N$ scalar fields into $N$ "kinetic fluids" with energy density and pressure, respectively,

$$
\rho_{I} \equiv \frac{1}{2} \dot{\varphi}_{I}^{2}, \quad P_{I} \equiv \frac{1}{2} \dot{\varphi}_{I}^{2}
$$

and a single "potential fluid" or vacuum energy,

$$
\rho_{U} \equiv U, \quad P_{U} \equiv-U .
$$

We now have

$$
\rho_{\varphi}=\rho_{U}+\sum_{I} \rho_{I}, \quad P_{\varphi}=P_{U}+\sum_{I} P_{I} .
$$

The kinetic and potential fluids have a barotropic equation of state and adiabatic sound speeds

$$
c_{\mathrm{s} I}^{2}=1, \quad c_{\mathrm{s} U}^{2}=-1
$$

The Klein-Gordon equation for each field,

$$
\ddot{\varphi}_{I}+3 H \dot{\varphi}_{I}+U_{, \varphi_{I}}=0
$$

gives a continuity equation for each kinetic energy (4.5) of the form given in Eq. (2.10), where the energy transfer to the kinetic fluids from the potential is given by

$$
Q_{I}=-\dot{\varphi}_{I} U_{, \varphi_{I}}
$$

where $U_{, \varphi_{I}} \equiv \partial U / \partial \varphi_{I}$. The constraint (2.5) then implies that the energy transfer to the potential is

$$
Q_{U}=\sum_{I} \dot{\varphi}_{I} U_{, \varphi_{I}}
$$

\section{B. Perturbations}

We now extend the fluid formalism to the perturbed scalar field case. 


\section{Standard treatment}

The total energy density and the pressure perturbation for $N$ scalar fields are

$$
\begin{aligned}
& \delta \rho_{\varphi}=\sum_{I}\left[\dot{\varphi}_{I} \dot{\delta \varphi_{I}}-\dot{\varphi}_{I}^{2} \phi+U_{, \varphi_{I}} \delta \varphi_{I}\right], \\
& \delta P_{\varphi}=\sum_{I}\left[\dot{\varphi}_{I} \dot{\delta} \varphi_{I}-\dot{\varphi}_{I}^{2} \phi-U_{, \varphi_{I}} \delta \varphi_{I}\right],
\end{aligned}
$$

having contributions both from the individual fields and the potential, whereas the total velocity perturbation, given by

$$
V_{\varphi}=-\frac{1}{\sum_{K} \dot{\varphi}_{K}^{2}} \sum_{I} \dot{\varphi}_{I} \delta \varphi_{I}
$$

is independent of the potential. Scalar fields cannot support anisotropic stress, and we have

$$
\Pi_{\varphi}=0 .
$$

The total non-adiabatic pressure perturbation for $N$ scalar fields is then readily calculated from Eq. (3.17) to be

$$
\delta P_{\mathrm{nad} \varphi}=\frac{2}{3 H \sum_{K} \dot{\varphi}_{K}^{2}} \sum_{I} \dot{\varphi}_{I}\left[\ddot{\varphi}_{I} \delta U-\dot{U}\left(\dot{\delta \varphi_{I}}-\dot{\varphi}_{I} \phi\right)\right]
$$

where

$$
\delta U \equiv \sum_{I} U_{, \varphi_{I}} \delta \varphi_{I}
$$

The total entropy perturbation for $N$ scalar fields was denoted $\mathcal{S}$ in Ref. [16] and is related to the above definition by

$$
\delta P_{\operatorname{nad} \varphi}=\frac{\dot{P}_{\varphi}}{H} \mathcal{S}
$$

The total entropy perturbation reduces in the single field case to

$$
\delta P_{\operatorname{nad} \varphi}=-\frac{2 U_{, \varphi} \ddot{\varphi}}{3 H}\left[\frac{\dot{\delta} \varphi-\dot{\varphi} \phi}{\ddot{\varphi}}-\frac{\delta \varphi}{\dot{\varphi}}\right]
$$

which is related to the intrinsic entropy perturbation $\Gamma$ defined in Ref. [30] by

$$
\delta P_{\operatorname{nad} \varphi}=\rho_{\varphi}\left(1-c_{\mathrm{s} \varphi}^{2}\right) \Gamma
$$

and to the intrinsic entropy perturbation $\Gamma_{\mathrm{q}}$ defined in Ref. [31] by $\delta P_{\operatorname{nad} \varphi}=P_{\varphi} \Gamma_{\mathrm{q}}$.

\section{Splitting into kinetic and potential parts}

Splitting the energy and pressure perturbation, as for the background fields, into $N$ "kinetic fluid" quantities and a single "potential fluid" we get

$$
\begin{aligned}
\delta \rho_{I} & =\dot{\varphi}_{I} \dot{\delta} \varphi_{I}-\dot{\varphi}_{I}^{2} \phi \\
\delta P_{I} & =\dot{\varphi}_{I} \dot{\delta} \varphi_{I}-\dot{\varphi}_{I}^{2} \phi \\
\Pi_{I} & =0
\end{aligned}
$$

for each kinetic fluid, and

$$
\delta \rho_{U}=\delta U=\sum_{I} U_{, \varphi_{I}} \delta \varphi_{I}, \quad \delta P_{U}=-\sum_{I} U_{, \varphi_{I}} \delta \varphi_{I}, \quad \Pi_{U}=0
$$


for the potential energy. We thus have $N$ gauge-invariant curvature perturbations, $\zeta_{I}$ defined according to Eq. (3.1), for each kinetic fluid plus one gauge-invariant curvature perturbation, $\zeta_{U}$, for the potential energy.

The covariant velocity perturbation for each kinetic fluid is

$$
V_{I}=-\frac{\delta \varphi_{I}}{\dot{\varphi}_{I}} .
$$

Substituting Eq. (4.24) into the definition of the total velocity, Eq. (2.19), we get the standard result, Eq. (4.13), for the total velocity perturbation due to the scalar fields. Note that the potential energy has no momentum perturbation and hence its covariant velocity perturbation, $V_{U}$, is undefined.

The comoving curvature perturbation, defined in Eq. (3.4), for each field is

$$
\mathcal{R}_{I}=\psi+H \frac{\delta \varphi_{I}}{\dot{\varphi}_{I}}
$$

and hence the total comoving curvature perturbation (i.e. relative to the average fluid velocity) is given by

$$
\mathcal{R}_{\varphi}=\frac{1}{\sum_{K} \dot{\varphi}_{K}^{2}} \sum_{I} \dot{\varphi}_{I}^{2} \mathcal{R}_{I}
$$

The Sasaki-Mukhanov variable [32, 33], or the field fluctuation on uniform curvature hypersurfaces, is simply $\mathcal{Q}_{I}=$ $\left(\dot{\varphi}_{I} / H\right) \mathcal{R}_{I}$.

Note that the field perturbations determine not only the velocity perturbations (4.24) but also the potential energy perturbation (4.23). Thus $\delta \rho_{U}$ and $V_{\varphi}$ are not independent variables. In terms of the gauge-invariant comoving curvature perturbations $\mathcal{R}_{I}$ we then have, using Eq. (A26),

$$
\zeta_{U}=-\frac{1}{\dot{U}} \sum_{K} U_{, \varphi_{K}} \dot{\varphi}_{K} \mathcal{R}_{K}
$$

which we can rewrite in terms of gauge-invariant total scalar field comoving curvature perturbation (4.26) and velocity perturbations (4.13) and 4.24)

$$
\zeta_{U}=-\mathcal{R}_{\varphi}+H \sum_{I} \frac{U_{, \varphi_{I}} \dot{\varphi}_{I}}{\dot{U}}\left(V_{I}-V_{\varphi}\right) .
$$

For a single scalar field we have simply $\zeta_{U}=-\mathcal{R}_{\varphi}$.

Although we have decomposed the total energy-momentum of the $N$ scalar fields into $N$ kinetic fluids interacting with one potential "fluid", the potential has no momentum perturbation and its density perturbation can be written in terms of the kinetic fluid velocities. Hence we only need the $N$ density perturbations and $N$ velocity perturbations to describe the perturbed energy-momentum of $N$ scalar fields.

We get the perturbed energy transfer to each field by substituting Eq. (4.20) for the fluid density into the perturbed energy conservation equation (2.15), and using the perturbed Klein-Gordon equation for each field,

$$
\ddot{\delta} \varphi_{I}+3 H \dot{\delta} \dot{\varphi}_{I}+\frac{k^{2}}{a^{2}}\left(\delta \varphi_{I}-\dot{\varphi}_{I} \sigma_{\mathrm{s}}\right)+\sum_{J} U_{, \varphi_{I} \varphi_{J}} \delta \varphi_{J}-\dot{\varphi}_{I}(\dot{\phi}+3 \dot{\psi})+2 U_{, \varphi_{I}} \phi=0
$$

which gives

$$
\delta Q_{I}=-U_{, \varphi_{I}}\left(\dot{\delta} \varphi_{I}-\dot{\varphi}_{I} \phi\right)-\dot{\varphi}_{I} \sum_{J} U_{, \varphi_{I} \varphi_{J}} \delta \varphi_{J}
$$

The perturbed energy transfer constraint, (2.14), then gives the energy transfer perturbation to the potential

$$
\delta Q_{U}=\dot{\delta} \dot{U}-\dot{U} \phi
$$

where

$$
\dot{\delta U}=\sum_{I, J}\left[\dot{\varphi}_{I} U_{, \varphi_{I} \varphi_{J}} \delta \varphi_{J}\right]+\sum_{I} U_{, \varphi_{I}} \dot{\delta}_{\varphi_{I}}
$$


Finally we identify the momentum transfer to each component. Substituting the expressions for the $I$-fluid quantities, $V_{I}, \delta P_{I}, \rho_{I}+P_{I}$ and $c_{I}^{2}$ of Section IVB2 into the momentum conservation equation (2.17) and, noting that $\Pi_{I}=0$, we obtain the momentum transfer perturbation to the $I$-fluid

$$
f_{I}=U_{, \varphi_{I}}\left(\delta \varphi_{I}+\dot{\varphi}_{I} V\right),
$$

in terms of the field variables or, using Eq. (3.26),

$$
f_{I}=Q_{I}\left(V_{I}-V\right)=-f_{\mathrm{rel}, \mathrm{I}} .
$$

Hence the total momentum transfer for each field, $f_{I}+f_{\text {rel,I, }}$, that appears, for instance, in Eq. (3.28), is zero.

As noted earlier the potential fluid has vanishing momentum, and from the momentum conservation equation (2.17) for the potential we require

$$
f_{U}=Q_{U}\left(V-V_{\varphi}\right),
$$

which is zero if there are no fluids present other than the scalar fields and $V=V_{\varphi}$. One can verify that Eq. (2.14) for the total momentum transfer is satisfied with $f_{U}+\sum_{I} f_{I}=0$.

\section{Relative perturbations}

Substituting the expression (4.20) for the density perturbation of each "kinetic fluid", and the background density (4.4), into the definition for the relative density perturbation, Eq. (3.13), we get

$$
\mathcal{S}_{I J}=-3 H\left[\frac{\dot{\delta \varphi_{I}}}{\ddot{\varphi}_{I}}-\frac{\dot{\delta \varphi_{J}}}{\ddot{\varphi}_{J}}-\phi\left(\frac{\dot{\varphi}_{I}}{\ddot{\varphi}_{I}}-\frac{\dot{\varphi}_{J}}{\ddot{\varphi}_{J}}\right)\right],
$$

which is, despite appearances, gauge-invariant as can be seen from the gauge transformation properties of the variables, Eqs. A33 and A18.

The relative density perturbation between each field and the potential is given by

$$
\mathcal{S}_{I U}=-3 H\left[\frac{\dot{\delta \varphi_{I}}-\dot{\varphi}_{I} \phi}{\ddot{\varphi}_{I}}-\frac{\delta U}{\dot{U}}\right] .
$$

However, remembering that the density perturbation for the potential can be written in terms of the field velocity perturbations, as in Eq. (4.28), we can also write the relative entropy perturbation, Eq. (3.13), between any fluid and the potential as

$$
\mathcal{S}_{\alpha U}=3\left(\zeta_{\alpha}+\mathcal{R}_{\varphi}\right)-3 H \sum_{I}\left(\frac{U_{, \varphi_{I}} \dot{\varphi}_{I}}{\dot{U}}\right) V_{I \varphi} .
$$

In the presence of additional fluids the total comoving curvature perturbation (3.6) is given by

$$
\mathcal{R}=\mathcal{R}_{\varphi}-H \sum_{\alpha} \frac{\rho_{\alpha}+P_{\alpha}}{\rho+P} V_{\alpha \varphi},
$$

and if we then use Eq. (3.7) to eliminate $\mathcal{R}$ in favour of $\zeta$ and $\Psi$, we finally obtain

$$
\mathcal{S}_{\alpha U}=\left(\frac{\dot{\rho}}{\dot{\rho}-\dot{U}}\right)\left[\frac{1}{\dot{H}} \frac{k^{2}}{a^{2}} \Psi+\sum_{\gamma \neq U} \frac{\dot{\rho}_{\gamma}}{\dot{\rho}} \mathcal{S}_{\alpha \gamma}-\frac{3 H}{\dot{U}} \sum_{K, \gamma} U_{, \varphi_{K}} \dot{\varphi}_{K} \frac{\rho_{\gamma}+P_{\gamma}}{\rho+P} V_{K \gamma}\right],
$$

where we also used Eq. A25.

The definition (4.36) of the relative entropy perturbation, $\mathcal{S}_{I J}$, between two fields in terms of their relative density perturbations, $\delta \rho_{I}$, differs from the definition used in Refs. [16, 20, 21], where the relative entropy perturbation was defined in terms of the relative field perturbations, $\delta \varphi_{I}$. In our fluid formalism the relative field perturbation is 
proportional to the relative velocity perturbation, defined in Eq. (3.16), which in the scalar field case, using Eq. (4.24), is given by

$$
V_{I J}=-\left(\frac{\delta \varphi_{I}}{\dot{\varphi}_{I}}-\frac{\delta \varphi_{J}}{\dot{\varphi}_{J}}\right)
$$

Indeed we can express the relative velocity perturbation, defined in Eq. (4.41), as the difference between comoving curvature perturbations,

$$
V_{I J}=-\frac{1}{H}\left(\mathcal{R}_{I}-\mathcal{R}_{J}\right)
$$

which is analogous to the definition of the relative entropy perturbation $\mathcal{S}_{\alpha \beta}$ expressed as the difference of curvature perturbations on uniform density hypersurfaces in Eq. (3.13). We shall see in a moment that when we evaluate the non-adiabatic energy transfer in terms of the relative perturbations between fields, the relative velocity perturbation (4.41) will indeed appear as a non-adiabatic perturbation.

Note that, as $V_{U}$ is not defined for the potential energy, the relative velocity perturbation, $V_{U \alpha}$, between any fluid and the potential energy is not defined. This would be a problem if $V_{U \alpha}$ appeared in the evolution equations (3.29 3.32) for our other fluid perturbations. However, $V_{U \alpha}$ only appears in summations over all fluids, $\gamma$, where it is multiplied by $\rho_{\gamma}+P_{\gamma}$, which vanishes for the potential energy.

\section{Non-adiabatic perturbations}

Each kinetic fluid and the potential fluid have definite equations of state $\left(\delta P_{I}=\delta \rho_{I}, \delta P_{U}=-\delta \rho_{U}\right)$. Hence, from (3.20), there is no intrinsic non-adiabatic pressure perturbation

$$
\delta P_{\text {intr }, \mathrm{I}}=0, \quad \delta P_{\text {intr, } \mathrm{U}}=0
$$

And since the kinetic fluids all have the same adiabatic sound speed $\left(c_{\mathrm{s} I}^{2}=1\right)$ there is no relative non-adiabatic pressure perturbation (3.22) due to entropy perturbations between the different kinetic fluids, $\mathcal{S}_{I J}$. The total entropy perturbation, Eq. (4.15), is thus due solely to the relative entropy perturbation between the fields and the potential (which has $c_{\mathrm{s} U}^{2}=-1$ ):

$$
\delta P_{\mathrm{nad}, \varphi}=\frac{2 \dot{U}}{9 H^{2} \sum_{K} \dot{\varphi}_{K}^{2}} \sum_{I} \dot{\varphi}_{I} \ddot{\varphi}_{I} \mathcal{S}_{I U}
$$

Note, however, that this entropy perturbation can always be re-expressed in terms of a sum over the other entropy perturbations and relative velocity perturbations, Eq. (4.38).

The intrinsic non-adiabatic energy transfer perturbation, defined in Eq. (3.23), for the I-fluids follows from Eqs. (4.4), (4.9), 4.20), and (4.30), and is given by

$$
\delta Q_{\mathrm{intr}, I}=\dot{\varphi}_{I}\left[\frac{\dot{U}_{, \varphi_{I}}}{\ddot{\varphi}_{I}}\left(\dot{\delta \varphi_{I}}-\dot{\varphi}_{I} \phi\right)-\delta U_{, \varphi_{I}}\right],
$$

which can be rewritten as

$$
\delta Q_{\mathrm{intr}, \mathrm{I}}=\sum_{J} \dot{\varphi}_{I} U_{, \varphi_{I} \varphi_{J}}\left[-\delta \varphi_{J}+\frac{\dot{\varphi}_{J}}{\ddot{\varphi}_{I}}\left(\dot{\delta \varphi_{I}}-\dot{\varphi}_{I} \phi\right)\right] .
$$

This is a relative entropy perturbation between the proper time derivative of the $I$-th field and the value of the $J$-th field. It is convenient to expand this as

$$
\delta Q_{\mathrm{intr}, I}=\sum_{J} \dot{\varphi}_{I} \dot{\varphi}_{J} U_{, \varphi_{I} \varphi_{J}}\left[\frac{\delta U}{\dot{U}}-\frac{\delta \varphi_{J}}{\dot{\varphi}_{J}}+\frac{\left(\dot{\delta \varphi_{I}}-\dot{\varphi}_{I} \phi\right)}{\ddot{\varphi}_{I}}-\frac{\delta U}{\dot{U}}\right],
$$

because, using Eq. (4.41), we can write

$$
\frac{\delta U}{\dot{U}}-\frac{\delta \varphi_{J}}{\dot{\varphi}_{J}}=\sum_{K} \frac{U_{, \varphi_{K}} \dot{\varphi}_{K}}{\dot{U}}\left[\frac{\delta \varphi_{K}}{\dot{\varphi}_{K}}-\frac{\delta \varphi_{J}}{\dot{\varphi}_{J}}\right]=\sum_{K} \frac{U_{, \varphi_{K}} \dot{\varphi}_{K}}{\dot{U}} V_{J K} .
$$


So finally, using Eq. (4.37), we can express the non-adiabatic intrinsic energy transfer in terms of the relative density perturbations $\mathcal{S}_{I U}$ and velocity perturbations $V_{I K}$

$$
\delta Q_{\mathrm{intr}, \mathrm{I}}=\sum_{J} \dot{\varphi}_{I} \dot{\varphi}_{J} U_{, \varphi_{I} \varphi_{J}}\left[\sum_{K} \frac{U_{, \varphi_{K}} \dot{\varphi}_{K}}{\dot{U}} V_{J K}-\frac{1}{3 H} \mathcal{S}_{I U}\right] .
$$

For the U-fluid the intrinsic non-adiabatic energy transfer perturbation is given by

$$
\delta Q_{\mathrm{intr}, \mathrm{U}}=\delta \dot{U}-\dot{U} \phi-\frac{\ddot{U}}{\dot{U}} \delta U,
$$

where we have used Eqs. (3.23), (4.5), (4.9), and (4.23), which yields in terms of relative entropy perturbations

$$
\delta Q_{\mathrm{intr}, \mathrm{U}}=-\frac{1}{3 H} \sum_{I} U_{, \varphi_{I}} \ddot{\varphi}_{I} \mathcal{S}_{I U}+\sum_{I, J} U_{, \varphi_{I} \varphi_{J}} \dot{\varphi}_{I} \dot{\varphi}_{J} \frac{\sum_{K} U_{, \varphi_{K}} \dot{\varphi}_{K}}{\dot{U}} V_{K J} .
$$

Thus we see that a non-zero relative velocity perturbation, $V_{J K}$, between scalar fields leads to a non-adiabatic energy transfer in Eqs. (4.49) and (4.51). To recover the adiabatic solution (3.38) on long-wavelengths with $\mathcal{S}_{I U}=0$ we see, from Eq. (3.36), that we require in addition that $V_{J K} \rightarrow 0$ for scalar fields with arbitrary interaction potential $U\left(\varphi_{I}\right)$. Thus we can identify the relative velocity perturbation $V_{J K}$ between scalar fields as a non-adiabatic perturbation [16], in contrast to the case of perfect fluids where we require only that $k^{2} V_{\alpha \beta} \rightarrow 0$ as $k \rightarrow 0$ to obtain an adiabatic solution with $\mathcal{S}_{\alpha \beta}=0$ on large scales.

We can finally rewrite the momentum transfer (4.34) solely in terms of a sum over the relative velocity perturbations $V_{I \alpha}$, according to Eq. (3.27),

$$
f_{I}=-\dot{\varphi}_{I} U_{, \varphi_{I}} \sum_{\alpha} \frac{\rho_{\alpha}+P_{\alpha}}{\rho+P} V_{I \alpha}
$$

\section{DISCUSSION AND CONCLUSIONS}

In this paper we have extended the analysis in the classic paper by Kodama and Sasaki 2] to deal with the coupled evolution of curvature and isocurvature perturbations in a multi-component cosmology with interacting fluids and scalar fields. In doing so we have clarified the nature of adiabatic initial conditions, and identified non-adiabatic effects.

It is well-known that the total curvature perturbation $\zeta$ is conserved on large scales when the total non-adiabatic pressure perturbation (3.17) vanishes. In a multi-component system the total non-adiabatic pressure perturbation can be split, according to Eq. (3.18), into the sum of the intrinsic non-adiabatic pressure perturbations of individual components and the relative pressure perturbation due to the relative density perturbations, $\mathcal{S}_{\alpha \beta}$, between different components. The intrinsic non-adiabatic pressure perturbations depend on the internal degrees of freedom of the fluid, but must vanish for fluids with a definite equation of state $P_{\alpha}\left(\rho_{\alpha}\right)$.

The relative density perturbation between different components, $\mathcal{S}_{\alpha \beta}$ defined in (3.13), generalises the perturbation in the photon-baryon ratio in a standard hot big bang cosmology to arbitrary interacting fluids. Hence we refer to $\mathcal{S}_{\alpha \beta}$ as a relative entropy (or isocurvature) perturbation.

We have given, for the first time, the evolution equations on all scales for the gauge-invariant entropy perturbations $\mathcal{S}_{\alpha \beta}$ in a multi-component system, which is driven by the intrinsic non-adiabatic pressure perturbations and perturbed energy transfer. Analogously to the pressure perturbations we split the perturbed energy transfer into the gauge-invariant intrinsic non-adiabatic energy transfer and the relative energy transfer, due to the relative entropy perturbations, $\mathcal{S}_{\alpha \beta}$. In certain models, such as spontaneous decay of non-relativistic particles [15], one may also be able to give the intrinsic non-adiabatic energy transfer in terms of the relative entropy perturbations, enabling one to obtain a closed set of evolution equations.

The overall curvature perturbation and relative entropy perturbations are also coupled to the velocity perturbations, but these decouple in the large scale limit (in the absence of intrinsic non-adiabatic pressure and intrinsic non-adiabatic energy transfer). Hence adiabatic density perturbations are characterised by a single amplitude, $\zeta$, which remains constant on large scales. A sufficient condition for adiabatic perturbations on large scales is

$$
\delta P_{\text {intr }, \alpha}=0, \quad \delta Q_{\text {intr }, \alpha}=0, \quad \text { and } \quad \mathcal{S}_{\alpha \beta}=0,
$$

for all components $\alpha$ and $\beta$. Adiabatic perturbations then stay adiabatic on large scales. 
We have also shown how to describe scalar fields and their perturbations in this notation. $N$ interacting scalar fields can be described by $N$ kinetic "fluids" with a stiff equation of state, $P_{I}=\rho_{I}$, interacting with one potential "fluid" with vacuum equation of state, $P_{U}=-\rho_{U}$. The potential fluid velocity is undefined, because its momentum perturbation $\left(\rho_{U}+P_{U}\right) V_{U}$ is zero. Because the components have fixed equations of state there are no intrinsic nonadiabatic pressure perturbations, only the relative non-adiabatic pressure due to relative entropy perturbations. On the other hand there is an intrinsic non-adiabatic energy transfer which includes terms due to the relative velocity perturbation, $V_{I J}$, even on large scales. Thus adiabatic perturbations for interacting scalar fields on large scales require

$$
S_{I J}=0, \quad S_{I U}=0, \quad \text { and } \quad V_{I J}=0 .
$$

Each scalar field perturbation $\delta \varphi_{I}$ determines the velocity perturbation, $V_{I}$, and thus adiabatic field perturbations [16] must obey

$$
V_{I J}=\frac{\delta \varphi_{J}}{\dot{\varphi}_{J}}-\frac{\delta \varphi_{I}}{\dot{\varphi}_{I}}=0
$$

During inflation scalar field perturbations originate as small-scale quantum fluctuations and these will not in general respect this adiabatic condition. On the other hand the existence of a unique attractor in phase space at late times would drive perturbations towards $V_{I J}=0$. In addition the adiabatic condition, $\mathcal{S}_{I J}=0$, for relative density perturbations requires that

$$
\frac{\delta \dot{\varphi}_{I \tau}}{\ddot{\varphi}_{I}}=\frac{\delta \dot{\varphi}_{J \tau}}{\ddot{\varphi}_{J}}
$$

where $\delta \dot{\varphi}_{I \tau} \equiv \dot{\delta} \dot{\varphi}_{I}-\dot{\varphi}_{I} \phi$ is the perturbed proper time derivative of the field, and $\mathcal{S}_{I U}=0$ requires

$$
\frac{\delta \dot{\varphi}_{I \tau}}{\ddot{\varphi}_{I}}=\frac{\delta U}{\dot{U}} .
$$

All of the above conditions can be enforced by requiring the generalised adiabatic condition for perturbations in any 4 -scalars $x$ and $y$ [10]:

$$
\frac{\delta x}{\dot{x}}=\frac{\delta y}{\dot{y}} .
$$

This is a generalisation of the usual adiabatic condition, $\mathcal{S}_{\alpha \beta}=0$, for fluid density perturbations which requires

$$
\frac{\delta \rho_{\alpha}}{\dot{\rho}_{\alpha}}=\frac{\delta \rho_{\beta}}{\dot{\rho}_{\beta}} .
$$

Because the scalar field perturbation determines both the velocity perturbation and the potential perturbation, $\mathcal{S}_{I U}$, is not independent of the other variables. For instance, for a single scalar field we have, from Eq. (4.40),

$$
\mathcal{S}_{I U}=\left(\frac{\dot{\rho}}{\dot{\rho}-\dot{U}}\right)\left[\frac{1}{\dot{H}} \frac{k^{2}}{a^{2}} \Psi+\sum_{\gamma \neq U}\left(\frac{\dot{\rho}_{\gamma}}{\dot{\rho}} \mathcal{S}_{I \gamma}-3 H \frac{\rho_{\gamma}+P_{\gamma}}{\rho+P} V_{I \gamma}\right)\right] .
$$

In a cosmology dominated by the scalar field this forces the scalar field perturbations to become adiabatic, $\mathcal{S}_{I U} \rightarrow 0$, in the $k \rightarrow 0$ limit for finite $\Psi$ [16]. But in the presence of other fluids, adiabatic field perturbations require the scalar field velocity to coincide with the total velocity perturbation, and hence $\sum_{\gamma}\left(\rho_{\gamma}+P_{\gamma}\right) V_{I \gamma}=0$.

Our formalism is applicable to a variety of cosmological models including interacting scalar fields and fluids. Although we have only considered minimally coupled scalar fields our formalism can naturally be extended to nonminimally coupled fields. Conformally transforming to the Einstein frame introduces interactions between a scalar field and the matter, of the form 34$]$

$$
Q_{(I)}^{\mu} \propto T_{m} \nabla^{\mu} \varphi_{I},
$$

where $T_{m}$ is the trace of the matter energy-momentum tensor. On the other hand the perturbed decay of an oscillating massive scalar field into light particles may be described by the perturbed energy transfer between pressureless matter and radiation [6]. 


\section{Acknowledgments}

The authors are grateful to David Lyth for useful comments. KAM is supported by PPARC grant PPA/G/S/2002/00098. DW is supported by the Royal Society.

\section{APPENDIX A: GAUGE TRANSFORMATIONS AND SOME USEFUL EQUATIONS}

\section{Gauge transformations}

The metric tensor, including linear scalar perturbations about a flat FRW background is given by

$$
g_{\mu \nu}=\left(\begin{array}{cc}
-1-2 \phi & a B_{, i} \\
a B_{, j} & a^{2}\left[(1-2 \psi) \dot{\delta}_{i j}+2 E_{, i j}\right]
\end{array}\right) .
$$

A first order coordinate transformation $\tilde{x}^{\mu}=x^{\mu}+\delta x^{\mu}$, where $\delta x^{\mu}=\left[\delta t, \delta x^{i}\right]$, induces a change in the metric tensor

$$
\widetilde{g_{\mu \nu}}=g_{\mu \nu}-£_{\delta x^{\mu}} g_{\mu \nu}
$$

where $£_{\delta x^{\mu}}$ denotes the Lie-derivative with respect to $\delta x^{\mu}$.

The scalar functions $\phi, \psi, B$ and $E$ change under the transformation as

$$
\begin{aligned}
\widetilde{\phi} & =\phi-\dot{\delta} t \\
\widetilde{\psi} & =\psi+\frac{\dot{a}}{a} \delta t, \\
a \widetilde{B} & =a B-a^{2} \dot{\delta} x+\delta t, \\
\widetilde{E} & =E-\delta x .
\end{aligned}
$$

The total four velocity is subject to the constraint

$$
u_{\mu} u^{\mu}=-1
$$

and allowing for linear perturbations we find

$$
\begin{aligned}
u_{\mu} & =\left[-(1+\phi), a(v+B)_{, i}\right], \\
u^{\mu} & =\left[1-\phi, \frac{1}{a} v^{i},\right]
\end{aligned}
$$

A first order coordinate transformation induces a change in the four velocity according to

$$
\widetilde{u}_{\mu}=u_{\mu}-£_{\delta x^{\mu}} u_{\mu}
$$

In a similar fashion we can define fluid four velocities for the individual fluids. We then find that the velocity potentials transform as

$$
\tilde{v}=v+a \dot{\delta x}, \quad \tilde{v}_{\alpha}=v_{\alpha}+a \dot{\delta x}
$$

and hence the covariant scalar velocity perturbations, defined above in Eqs. (2.16) and (2.20), as

$$
\begin{aligned}
V_{\alpha} & \equiv a\left(v_{\alpha}+B\right) \\
V & \equiv a(v+B)
\end{aligned}
$$

transform as

$$
\begin{aligned}
\widetilde{V_{\alpha}} & =V_{\alpha}+\delta t, \\
\widetilde{V} & =V+\delta t .
\end{aligned}
$$

Note, in [15] we used momentum perturbations $\delta q$ and $\delta q_{\alpha}$ instead of the velocity perturbations $V$ and $V_{\alpha}$ which are related by

$$
\begin{aligned}
\delta q_{\alpha} & =\left(\rho_{\alpha}+P_{\alpha}\right) V_{\alpha} \\
\delta q & =(\rho+P) V
\end{aligned}
$$


The total and the individual momenta change as

$$
\widetilde{\delta q}=\delta q+(\rho+P) \delta t, \quad \widetilde{\delta q_{\alpha}}=\delta q_{\alpha}+\left(\rho_{\alpha}+P_{\alpha}\right) \delta t .
$$

The shear scalar, $\sigma_{\mathrm{s}} \equiv a^{2} \dot{E}-a B$, changes as

$$
\tilde{\sigma}_{\mathrm{s}}=\sigma_{\mathrm{s}}-\delta t
$$

The density perturbation changes as

$$
\widetilde{\delta \rho}=\delta \rho-\dot{\rho} \delta t .
$$

The perturbed energy transfer four vector of the $\alpha$-fluid, $Q_{(\alpha)}^{\nu}$, defined in Eq. (2.13), transforms as

$$
\widetilde{Q}_{(\alpha)}^{\nu}=Q_{(\alpha)}^{\nu}-£_{\delta x^{\mu}} Q_{(\alpha)}^{\nu},
$$

and we find that the perturbed energy transfer to the $\alpha$-fluid changes as

$$
\widetilde{\delta Q_{\alpha}}=\delta Q_{\alpha}-\dot{Q}_{\alpha} \delta t
$$

whereas the perturbed momentum transfer is gauge-invariant

$$
\widetilde{f}_{i}=f_{i}
$$

\section{Identities}

a. Background

A useful relation between the Hubble parameter and the background energy density can be derived by combining Eqs. (2.6), (2.8), and (2.7) to give

$$
\frac{\dot{H}}{H}=\frac{\dot{\rho}}{2 \rho}
$$

\section{b. Perturbations}

In order to derive the set of evolution equations for $\mathcal{S}_{\alpha \beta}$ and $V_{\alpha \beta}$, Eqs. (3.31) and (3.32), we made use of the following relations between the $\alpha$-fluid and total density perturbation and the relative entropy perturbation,

$$
\frac{\delta \rho_{\alpha}}{\dot{\rho}_{\alpha}}=\frac{\delta \rho}{\dot{\rho}}-\frac{1}{3 H} \sum_{\gamma} \frac{\dot{\rho}_{\gamma}}{\dot{\rho}} \mathcal{S}_{\alpha \gamma},
$$

and the relation between the relative velocity perturbation and the $\alpha$-fluid and total velocity perturbation,

$$
V_{\alpha}=V+\sum_{\beta} \frac{\rho_{\beta}+P_{\beta}}{\rho+P} V_{\alpha \beta} .
$$

In terms of the curvature perturbations on uniform density and comoving hypersurfaces the above expressions give

$$
\begin{aligned}
\zeta_{\alpha} & =\zeta+\frac{1}{3} \sum_{\gamma} \frac{\dot{\rho}_{\gamma}}{\dot{\rho}} \mathcal{S}_{\alpha \gamma}, \\
\mathcal{R}_{\alpha} & =\mathcal{R}-H \sum_{\beta} \frac{\rho_{\beta}+P_{\beta}}{\rho+P} V_{\alpha \beta} .
\end{aligned}
$$

We found it useful to "symmetrise" some expressions involving differences in the $V_{\alpha \beta}$ :

$$
x_{\alpha} \sum_{\gamma} \frac{\rho_{\gamma}+P_{\gamma}}{\rho+P} V_{\alpha \gamma}-x_{\beta} \sum_{\gamma} \frac{\rho_{\gamma}+P_{\gamma}}{\rho+P} V_{\beta \gamma} \equiv \frac{1}{2}\left(x_{\alpha}+x_{\beta}\right) V_{\alpha \beta}+\frac{1}{2}\left(x_{\alpha}-x_{\beta}\right) \sum_{\gamma} \frac{\rho_{\gamma}+P_{\gamma}}{\rho+P}\left(V_{\alpha \gamma}+V_{\beta \gamma}\right) .
$$

A similar expression holds for $\mathcal{S}_{\alpha \beta}$, replacing the $\rho_{\gamma}+P_{\gamma}$ and $V_{\alpha \beta}$ by $\dot{\rho}_{\gamma}$ and $\mathcal{S}_{\alpha \beta}$. 


\section{APPENDIX B: EVOLUTION EQUATIONS IN THE UNIFORM CURVATURE GAUGE}

Uniform-density or uniform-field gauges, used to define the curvature perturbations $\zeta_{\alpha}$ and $\mathcal{R}_{I}$, can become illdefined in some cases even though nothing singular is happening in other gauges. This occurs whenever the uniform field hypersurfaces become singular, i.e. if $\dot{\varphi}_{I}=0$, as can be seen from Eq. (4.25) 35, 36], or when the uniform density hypersurfaces become singular, which happens when $\dot{\rho}_{\alpha}=0$, as can be seen from Eq. (3.1). In the latter context the equations presented below were used in [37], but only in their large scale limit. The singular behaviour can be eliminated by simply working with rescaled perturbation variables which physically corresponds to working with perturbations in the uniform-curvature gauge [2]. The density contrast $\Delta_{\mathrm{g}}$ defined in [2] and denoted $\Delta$ in [31] is related to the density perturbation on flat slices by $\Delta_{\mathrm{g}}=\Delta=\widetilde{\delta} \rho / \rho$.

Note that all the variables in this section are defined on uniform curvature hypersurfaces. To avoid confusion we denote quantities evaluated in the uniform curvature gauge by a "tilde". The curvature perturbations $\zeta, \zeta_{\alpha}, \mathcal{R}$ and $\mathcal{R}_{\alpha}$ are simply related to the density and velocity perturbations in the uniform-curvature gauge via Eqs. (3.11) and (3.12) .

In the case of multiple fluids the energy evolution equation of the $\alpha$-fluid in the uniform curvature gauge is given from Eq. (2.15) by

$$
\widetilde{\tilde{\delta \rho_{\alpha}}}+\left[3 H\left(1+c_{\alpha}^{2}\right)-\frac{\dot{Q}_{\alpha}}{\dot{\rho}_{\alpha}}\right] \widetilde{\delta \rho_{\alpha}}-\delta Q_{\mathrm{intr}, \alpha}+\delta P_{\mathrm{intr}, \alpha}-\frac{k^{2}}{a^{2}}\left(\rho_{\alpha}+P_{\alpha}\right)\left(\widetilde{V_{\alpha}}+\widetilde{\sigma}_{\mathrm{s}}\right)-Q_{\alpha} \frac{\dot{H}}{H} \widetilde{V}=0 .
$$

The evolution equation for the velocity perturbation of the $\alpha$-fluid, $V_{\alpha}$, is, using Eq. (2.17)

$$
\dot{V_{\alpha}}+\left[\frac{Q_{\alpha}}{\rho_{\alpha}+P_{\alpha}}\left(1+c_{\alpha}^{2}\right)-3 H c_{\alpha}^{2}\right] \widetilde{V_{\alpha}}+\frac{\dot{H}}{H} \widetilde{V}+\frac{1}{\rho_{\alpha}+P_{\alpha}}\left[\delta P_{\text {intr }, \alpha}+c_{\alpha}^{2} \widetilde{\delta \rho_{\alpha}}-\frac{2}{3} \frac{k^{2}}{a^{2}} \Pi_{\alpha}-Q_{\alpha} \widetilde{V}-f_{\alpha}\right]=0 .
$$

The total velocity and the velocities of the individual species are related by Eq. (2.19), whereas the total density perturbation is just the sum of the density perturbations of the individual fluids, Eq. (2.18). Summing over the evolution equations of the individual fluids, Eq. (B1), and using the constraints Eqs. (2.18) and (2.19), we find the total energy evolution equation to be

$$
\dot{\tilde{\delta}}+3 H\left(1+c_{\mathrm{s}}^{2}\right) \widetilde{\delta \rho}+3 H \delta P_{\text {nad }}-\frac{k^{2}}{a^{2}}\left[(\rho+P)\left(\widetilde{V}+\widetilde{\sigma}_{\mathrm{s}}\right)\right]=0,
$$

and the total momentum evolution equation from Eq. (B2),

$$
\dot{\vec{V}}+\left(\frac{\dot{H}}{H}-3 H c_{\mathrm{s}}^{2}\right) \widetilde{V}+\frac{c_{\mathrm{s}}^{2}}{\rho+P} \widetilde{\delta \rho}+\frac{1}{\rho+P}\left(\delta P_{\mathrm{nad}}-\frac{2}{3} \frac{k^{2}}{a^{2}} \Pi\right)=0 .
$$

The shear in the uniform curvature gauge, $\tilde{\sigma}$, is simply the rescaled metric potential $\Psi$, defined in Eq. (3.8), as can be seen from Eq. (3.11), so to close the system of equations we can either use the constraint equation (3.7) together with Eq. (3.11), or use the shear evolution equation in the uniform curvature gauge,

$$
\dot{\widetilde{\sigma_{\mathrm{s}}}}+H \widetilde{\sigma_{\mathrm{s}}}-\frac{\dot{H}}{H} \widetilde{V}-8 \pi G \Pi=0 .
$$

[1] J. M. Bardeen, Phys. Rev. D 22 (1980) 1882.

[2] H. Kodama and M. Sasaki, Prog. Theor. Phys. Suppl. 78 (1984) 1.

[3] V. F. Mukhanov, H. A. Feldman and R. H. Brandenberger, Phys. Rept. 215 (1992) 203.

[4] G. F. R. Ellis and M. Bruni, Phys. Rev. D 40, 1804 (1989). P. K. S. Dunsby, M. Bruni and G. F. R. Ellis, Astrophys. J. 395, 54 (1992).

[5] A. Challinor and A. Lasenby, Phys. Rev. D 58, 023001 (1998) arXiv:astro-ph/9804150.

[6] M. Bastero-Gil, V. Di Clemente and S. F. King, Phys. Rev. D 67, 103516 (2003) arXiv:hep-ph/0211011; G. Dvali, A. Gruzinov and M. Zaldarriaga, Phys. Rev. D 69, 023505 (2004) arXiv:astro-ph/0303591; L. Kofman, arXiv:astro-ph/0303614.

[7] K. Enqvist and M. S. Sloth, Nucl. Phys. B 626, 395 (2002) arXiv:hep-ph/0109214; D. H. Lyth and D. Wands, Phys. Lett. B 524, 5 (2002) arXiv:hep-ph/0110002; T. Moroi and T. Takahashi, Phys. Lett. B 522, 215 (2001) [Erratum-ibid. B 539, 303 (2002)] arXiv:hep-ph/0110096; Phys. Rev. D 66, 063501 (2002) arXiv:hep-ph/0206026; D. H. Lyth, C. Ungarelli and D. Wands, Phys. Rev. D 67, 023503 (2003) arXiv:astro-ph/0208055. 
[8] C. Gordon and W. Hu, Phys. Rev. D 70, 083003 (2004) arXiv:astro-ph/0406496.

[9] D. H. Lyth and D. Wands, Phys. Rev. D 68, 103515 (2003) arXiv:astro-ph/0306498.

[10] D. Wands, K. A. Malik, D. H. Lyth and A. R. Liddle, Phys. Rev. D 62 (2000) 043527 astro-ph/0003278.

[11] J. M. Bardeen, P. J. Steinhardt and M. S. Turner, Phys. Rev. D 28, 679 (1983).

[12] J. M. Bardeen, DOE/ER/40423-01-C8 Lectures given at 2nd Guo Shou-jing Summer School on Particle Physics and Cosmology, Nanjing, China, Jul 1988.

[13] D. Langlois, Phys. Rev. D 59, 123512 (1999) arXiv:astro-ph/9906080.

[14] T. Hamazaki and H. Kodama, Prog. Theor. Phys. 96 (1996) 1123 arXiv:gr-qc/9609036.

[15] K. A. Malik, D. Wands and C. Ungarelli, Phys. Rev. D 67 (2003) 063516 arXiv:astro-ph/0211602.

[16] C. Gordon, D. Wands, B. A. Bassett and R. Maartens, Phys. Rev. D 63 (2001) 023506 arXiv:astro-ph/0009131.

[17] A. Notari and A. Riotto, Nucl. Phys. B 644, 371 (2002) arXiv:hep-th/0205019.

[18] F. Di Marco, F. Finelli and R. Brandenberger, arXiv:astro-ph/0211276 .

[19] J. c. Hwang and H. Noh, Phys. Lett. B 495, 277 (2000) arXiv:astro-ph/0009268.

[20] B. J. van Tent and S. Groot Nibbelink, arXiv:hep-ph/0111370.

[21] G. Rigopoulos, Class. Quant. Grav. 21, 1737 (2004) arXiv:astro-ph/0212141.

[22] J. c. Hwang and H. Noh, Class. Quant. Grav. 19, 527 (2002) arXiv:astro-ph/0103244. Phys. Rev. D 64 (2001) 103509 arXiv:astro-ph/0108197.

[23] Y. Nambu and A. Taruya, Class. Quant. Grav. 15, 2761 (1998) arXiv:gr-qc/9801021]; Y. Nambu and S. i. Ohokata, Class. Quant. Grav. 19, 4263 (2002) arXiv:gr-qc/0207019.

[24] K. A. Malik and D. Wands, in preparation.

[25] K. A. Malik, arXiv:astro-ph/0101563.

[26] V. N. Lukash, Sov. Phys. JETP 52(5), 807 (1980) [Zh. Eksp. Teor. Fiz. 79, 1601 (1980)].

[27] D. H. Lyth, Phys. Rev. D 31 (1985) 1792.

[28] J. Garcia-Bellido and D. Wands, Phys. Rev. D 53, 5437 (1996), arXiv:astro-ph/9511029.

[29] D. H. Lyth, K. A. Malik and M. Sasaki, arXiv:astro-ph/0411220 .

[30] M. Malquarti and A. R. Liddle, Phys. Rev. D 66, 123506 (2002) arXiv:astro-ph/0208562 ; N. Bartolo, P. S. Corasaniti, A. R. Liddle and M. Malquarti, Phys. Rev. D 70, 043532 (2004) arXiv:astro-ph/0311503.

[31] M. Doran, C. M. Muller, G. Schafer and C. Wetterich, Phys. Rev. D 68, 063505 (2003) arXiv:astro-ph/0304212 .

[32] M. Sasaki, Prog. Theor. Phys. 76, 1036 (1986).

[33] V. F. Mukhanov, Sov. Phys. JETP 67, 1297 (1988) [Zh. Eksp. Teor. Fiz. 94N7, 1 (1988)].

[34] T. Damour and G. Esposito-Farese, Class. Quant. Grav. 9, 2093 (1992).

[35] A. R. Liddle, D. H. Lyth, K. A. Malik and D. Wands, Phys. Rev. D 61, 103509 (2000) arXiv:hep-ph/9912473.

[36] F. Finelli and R. H. Brandenberger, Phys. Rev. Lett. 82, 1362 (1999) arXiv:hep-ph/9809490.

[37] S. Gupta, K. A. Malik and D. Wands, Phys. Rev. D 69, 063513 (2004) arXiv:astro-ph/0311562. 